\title{
DEEP LEARNING AND GLOBALLY GUIDED IMAGE FILTERING TECHNIQUE BASED IMAGE DEHAZING AND ENHANCEMENT
}

\author{
Sangeeta Rathi ${ }^{1}$, Savita Bamal ${ }^{2}$ \\ E-Mail Id: ${ }^{1}$ sangitarathi1321@gmail.com, ${ }^{2}$ savitabamal85@gmail.com
}

Department of Computer Science, DPG Institute of Technology \& Management, Gurugram, Haryana

Abstract: Haze in images is due to natural environmental phenomena, which makes the image in a white shade noise. Haze removal is one of the most important research topics these days due to popularity of applications in the real surveillance from drones or any area under security. Both indoor and outdoor images are important for testing haze and its removal. Many image processing techniques are made by researchers to remove haze in a single image. Haze intensity can be calculated by a parameter known as perceptual fog density (PFD). It is important to analyze this parameter for all the techniques so as to get an idea of improvement. In this paper, a new approach is made by applying globally guided filtering technique with deep neural network. This proposed algorithm is implemented on MATLAB software and results are obtained by calculating PFD in the existing and proposed technique. The four techniques are compared with each other. The techniques are global filtering (GIF), weighted global filtering (WGIF), globally guided filtering (GGIF) and proposed technique i.e. globally guided filtering with DNN (Deep Neural Network). In GIF, the fine structure of the image is generally not preserved, but in proposed algorithm, the PDF is the minimum with fine structure, color intensity of the picture is of the best quality.

Keywords: Dehazing, GGIF, Deep Learning, Machine Learning; PFD.

\section{INTRODUCTION}

The light reflected from one object stretches in the atmosphere before landing on the screen in almost every useful scenario. [1] This is because there are aerosols like dirt, mist and gases which blocks light from its initial spread. The picture in which the highlights are diminished and ground colors becomes the weak in long distance photography and foggy condition has a significant effects. [2] These deteriorated photos frequently lack graphical and attractive images, and in addition the scene material provides poor visibility. This effect can be of disgust and can weaken the value of under-water and aerial photography for amateur, industrial and creative photographers. It may also be used for satellite imagery, including geological and cloud-based mapping, land planning and architecture design. [3] The result is a cumulative loss of picture contrast and an additive term because of this uniform light. The template used in the presence of haze to formalize image creation. [4] The degraded picture is interpreted in this model as a total of two components: the contribution of air light and the uncertain radiance of the ground. The transmission rate, a scalar that sets the visibility at each pixel, combines the two three channel color vectors algebraically. The surface color values and the transfer value for each pixel must be determined in order to recover a hazel free. [5]

The process is uncertain and cannot evaluate the transmitting quality because the source picture accounts for three formulas a pixel. Because the question cannot respond on the basis of one single image is: look over a thick white layer on a deep red surface and see a soft red surface on a similar or translucent medium. [6] A new way to recover an unspoilt image received as an input with single photograph in this study. The picture is split into the areas of a constant albedo, and the uncertainty of air light-albedo is solved by creating an additional restriction that needs a regional statistical correlation of superficial shading and media transmitting functions. [7] This requires a significant difference between the shading element and the noise in the picture. [8] The correlation theory is also used to measure the hue of the air sun. This approach is passive: numerous scene frames, light blocking distortion, data regarding landscape distance, or advanced sensor or equipment are no longer necessary. It also does not suppose that the hazel surfaces are smooth in place, i.e. scene depth and 
medium thickness discontinuities are needed.

\section{LITERATURE REVIEW}

Zhengguo Li et al., Fine structure, such as managed image filtering (GIF) and weighted object orientation (WGIF), cannot be preserved by local edge preservation techniques for smoothing. This filter will be used to identify individual images, demonstrating that G-GIF merely retains the fine structure of a dehazed pictorial photograph and that the dehazing G-GIF is smoother than that seen in existing GIF dehazed photos. The G-GIF provides a global boundary protection smoothing filter and a regional structure conversion feature. [1]

Dengyin Zhang et al., Haze retrieves the hazy image phase into a hazy usable picture. On the basis of the cutting-edge protection strategy the distribution chart of a haze pictures is predicted. The weighted guidance image filter is applied to the directed object filter by applying edge aware weighting. The total color channel and dark channel of the haze image was determined. The dark stream is broken down into the base layer and the information sheet with the weighted directed picture filter. The transformation diagram is centered on the base layer. The chart is used to restore the image without hazelnut. [2]

Zheqi Lin et al., Poor visibility in bad weather, including mist or fog, are major challenges for many computer vision applications. Highly necessary for the quality of the graphical algorithm is the elimination of haze. A new quick dehazing approach for video and image processing in real time. An improved guidance sorting scheme calculates the transmitting chart seamlessly and values the underlying picture profound data. The results demonstrate that both the dehazement and the real-time output of the system are great. The algorithm can be used for beneficial preprocessing in many applications, including security, smart vehicles and remote sensing because of its pace and visual enhancement performance. The results show that both dehazement and system's real-time efficiency were good. Thanks to their pace and visual enhancement, the device algorithm can be used for useful preprocessing on many applications including security, smart vehicles and remotely sensing. [3]

He Zhang et al., Our approach tries to recover the dehazed picture accurately instead of calculating first the transmission map, in comparison to the existing methods. The method involves an encoder-decoder design that integrates background knowledge in the decoder when decoding with a pyramid pooling unit. The network is discovered by increasing square error and expected failure to the standard. During the learning and inference process, multi-scale patches are used to further improve performance. [4]

Jiahao Pang et al., The dehazing of a single image is complicated because it is very ugly. The elimination of haze based on dark channel prior is successful but it is computationally expensive to refine the transmission map. Recent work has shown that it is possible to use a directed filter to simplify the map. Through merging the dark channel with the controlled object filtering in depth, we are producing single image dehazing. An active method of adjusting criteria, by evaluating the trade off of the solution. Our system produces satisfactorily dehazed tests at low approximation, as shown in experiments and correlations. [5]

\section{DISCUSSION}

A new globally guided image filtering is introduced in this paper. The proposed filter can be applied to produce sharper images and preserves details in regions of fine structure visibly better than the existing locally guided image filtering. It is applied to study single image haze removal. Experimental results demonstrate that the proposed haze removal algorithm indeed improves visual quality of dehazed images. Besides single image haze removal, there are many applications of the proposed filters. For example, the filter can be applied to study panorama imaging, edge-aware smoothing pyramid for exposure fusion, detail enhancement image matting, HDR compression, feathering, high resolution up-sampling, and so on. We will study application in our future research.

\section{IMPLEMENTATION}

In this section, the proposed algorithm implementation and results are presented for the GGIF and DNN proposed algorithm, firstly, the GGIF algorithm will be explained in brief here, and then the DNN part will be explored in the following section. Several input images are taken into consideration for this paper, which are shown in the Fig. 4.1.

In this section, proposed algorithm is discussed thesoritically for image dehazing method. This new method consists of two phases: 
$>$ Globally guided image filtering (GGIF)

$>$ Deep Neural Network (DNN)

\subsection{Globally Guided Image Filtering (GGIF)}

GGIF method is a mathematical equation based filtering method which is applied with global filter and a WLS filter is used.

\subsection{Deep Neural Network (DNN)}

DNN is also known as Deep Learning. In DNN, a haze free image is already trained in the data base, then according to matching similarity the haze free images are tested according to intensity variable values.
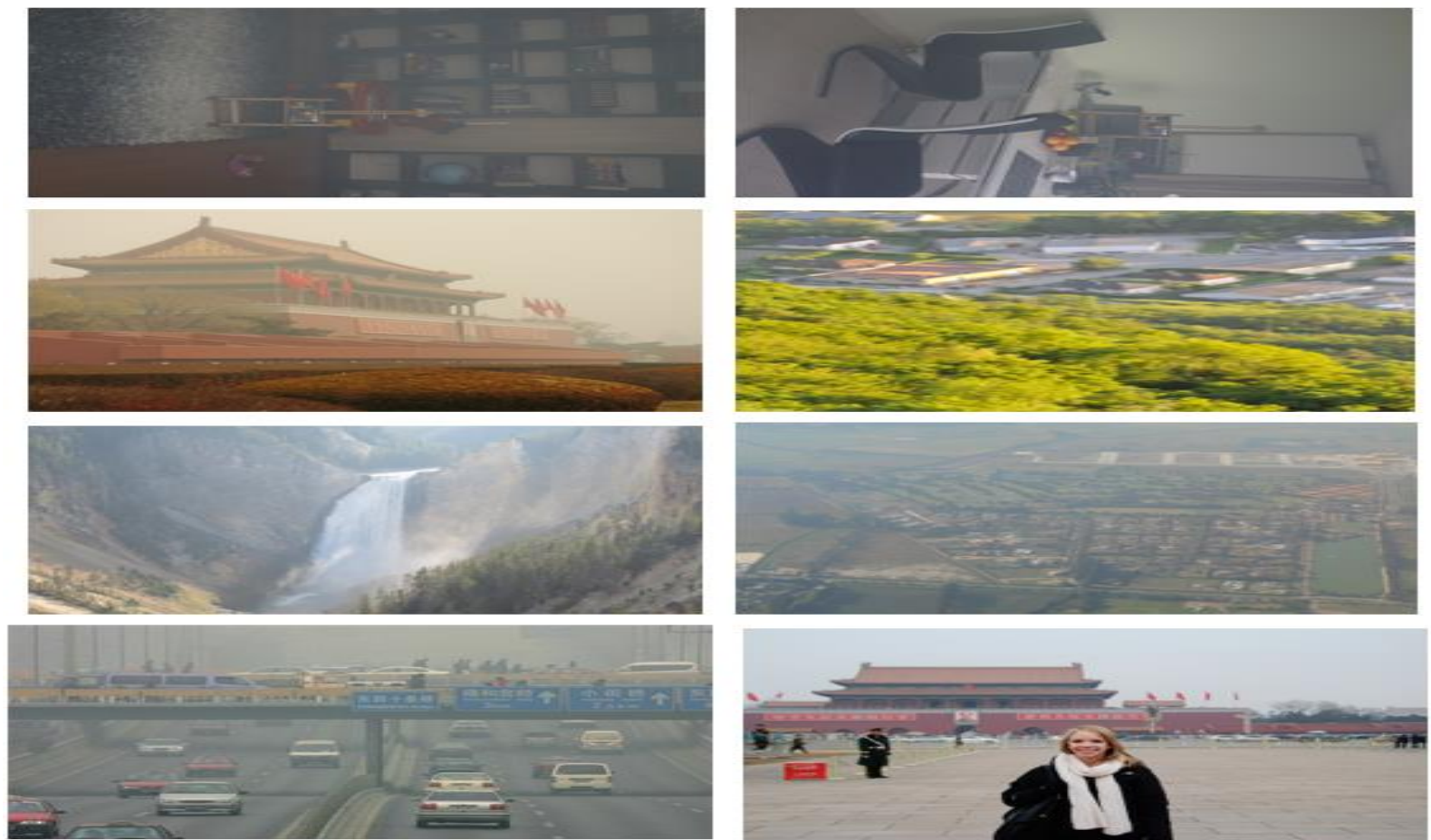

Fig. 4.1 Input Images for Implementation

Not at all like the GIF in figure 1 and the WGIF in figure 1, the proposed channel is a worldwide channel and it is therefore called the G-GIF. Contribution of the proposed G-GIF are a picture to be sifted and a direction vector field while contributions of the GIF and WGIF are a picture to contract data ought to be extraordinary be separated and a direction picture. The structure is characterized by the direction vector field. The proposed G-GIF is made out of a worldwide structure move channel and a worldwide edge-safeguarding smoothing channel. The capacity of the structure move channel is to move the predefined structure to the picture to be separated while the capacity of the smoothing channel is to smooth the moved picture in order to create the yield picture. In G-GIF, a box filter is created by taking values of sigma, mean and variance using basic formulae, which is then applied to G-GIF edge preserving algorithm. The factors into consideration of an image are covariance, mean, variance, mean of variance known as alpha, which are then given to the following equation 1 .

$$
\mathrm{q}=\text { mean } \_\mathrm{a} * \mathrm{I}+\text { mean_b... }
$$

The output $\mathrm{q}$ is then fed to WLS filter for further processing of G-GIF technique. The final G-GIF output is then passed through the Deep Neural Network.

In WLS filter the alpha, mean and variance are passes through a set of equations which are basically a mathematical Laplacian functions. A straightforward dimness picture model is determined by utilizing the disentangled dim channels of the standardized murkiness picture. From a factual perspective, if the two examples are close or comparative, i.e., the separation between them is little or the closeness is high, they give little data; the distinction data ought to be extraordinary.

$\mathrm{DNN}$, a trainable start to finish framework that unequically learns the mapping relations between crude foggy pictures and their related medium transmission maps. In this segment, present layer plans of DNN, and talk about how these plans are identified with thoughts in existing picture dehazing strategies. The proposed DNN comprises of fell convolutional and a pooling combination layers, with proper nonlinear actuation capacities 
utilized after a portion of these layers. In this, firstly the white balance image is created; dehazing parameters trained are loaded, passed through nnconv and nnpool layers. The features are extracted through multi mapping local extremum calculations. The nnloss layer is applied, after which softmax layer is applied, proceeding with relu layer and offset layer for neural networks in image dehazing context. Then normalizing of layers is taken into considerations. The final layer output image is saved for checking the perceptual fog density. Flowchart of implementation is shown below in fiG. 4.2.

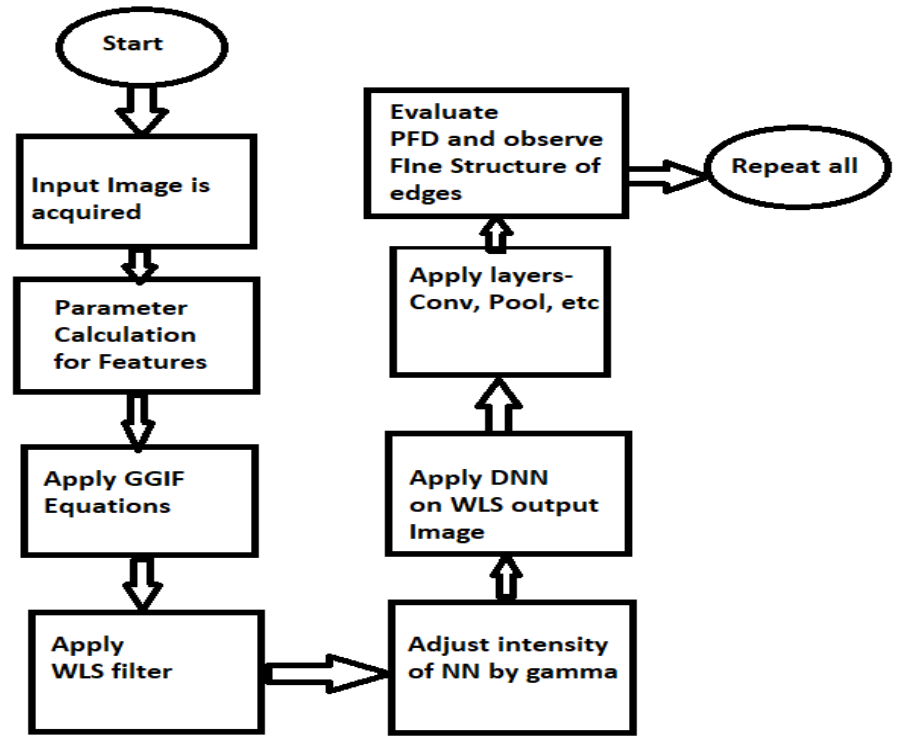

Fig. 4.2 Flowchart of Proposed Algorithm

\section{RESULT}

In this section, the results of all images are seen on the basis of PFD and its edge preserving and fine structure of the colors are observed.

In Fig. 5.1, the GUI of first image is shown, and on right side it shows the PFD calculation of different images.

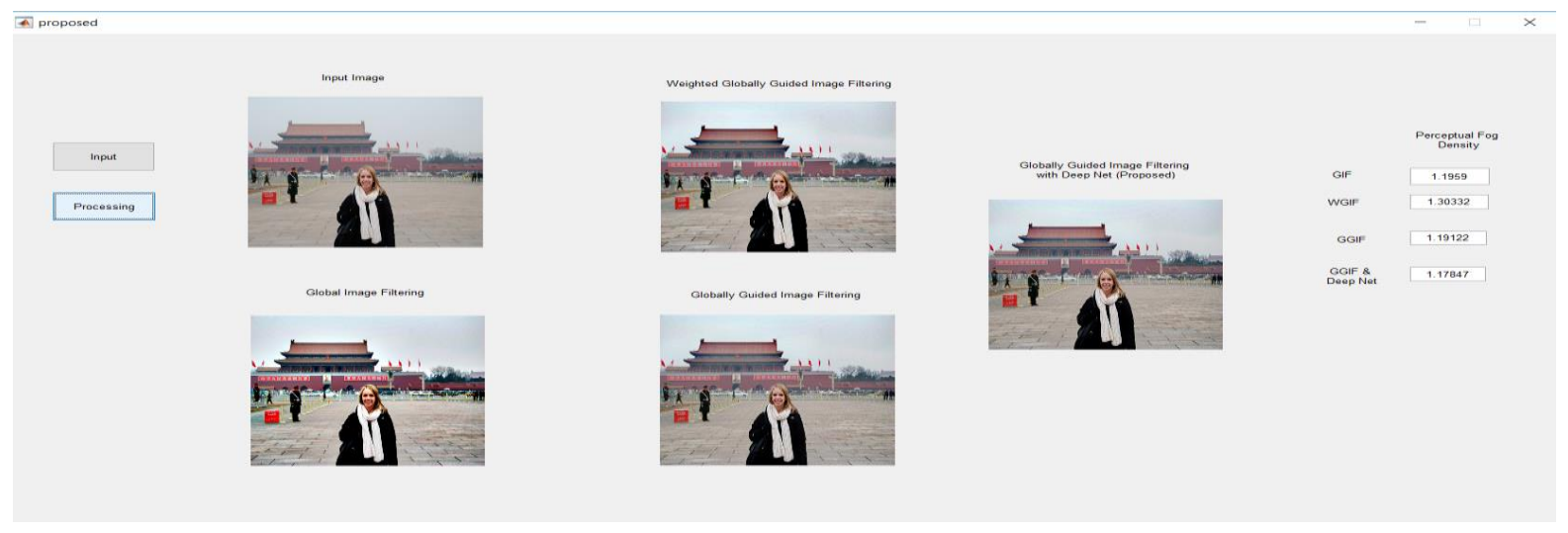

Fig. 5.1 Input Image 1 Output

In fig. 5.1, the fine structure is good in proposed and the PFD is lowest as shown in fig. 5.2.

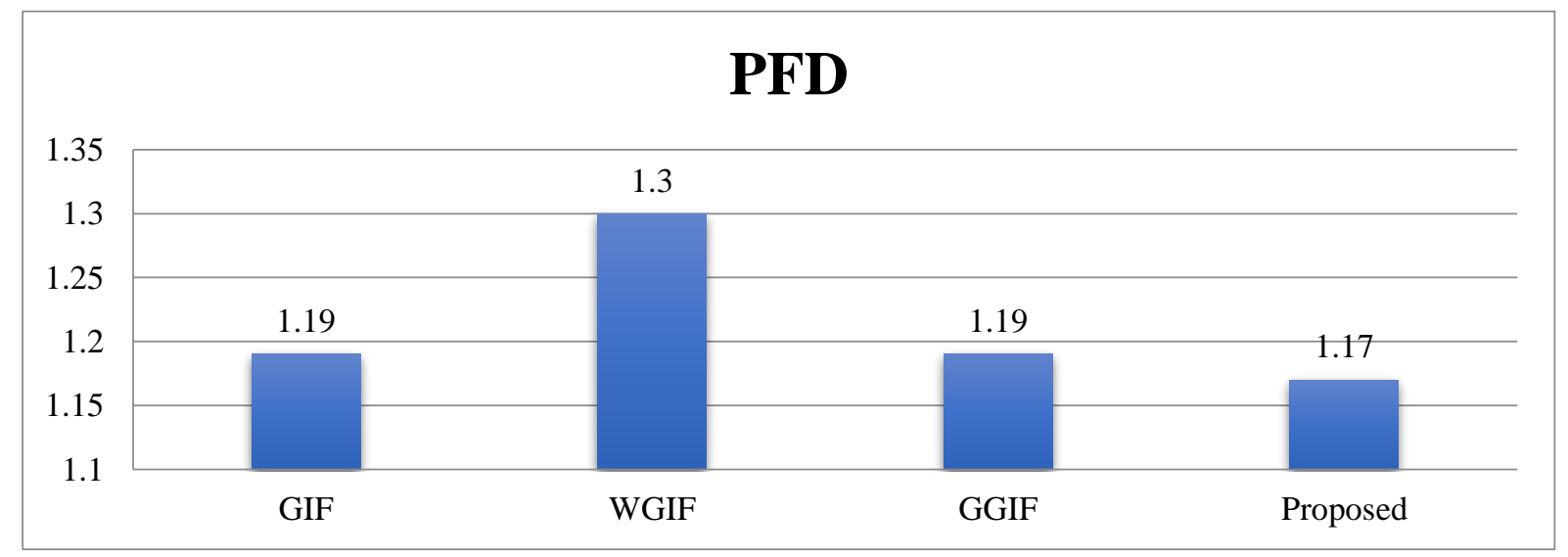

Fig. 5.2 Input Image 1 PFD Output 
ICACCG2020 30-31 July, 2020, Ansal University, Gurgaon, India

International Journal of Technical Research \& Science (Special Issue)

In fig. 5.2, the fine structure is good in proposed and the PFD is lower in case of proposed algorithm but similar to GIF and GIF does not have good color structure as shown in fig. 5.3.

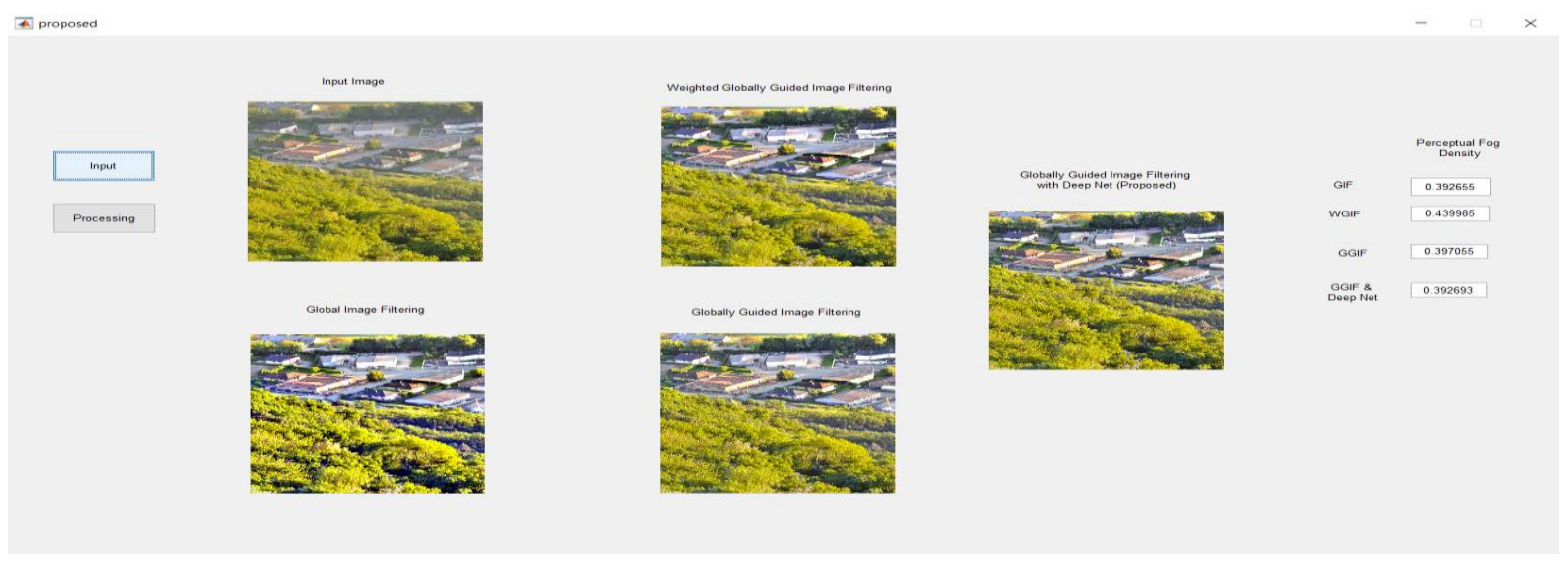

Fig. 5.3 Input Image 2 Output

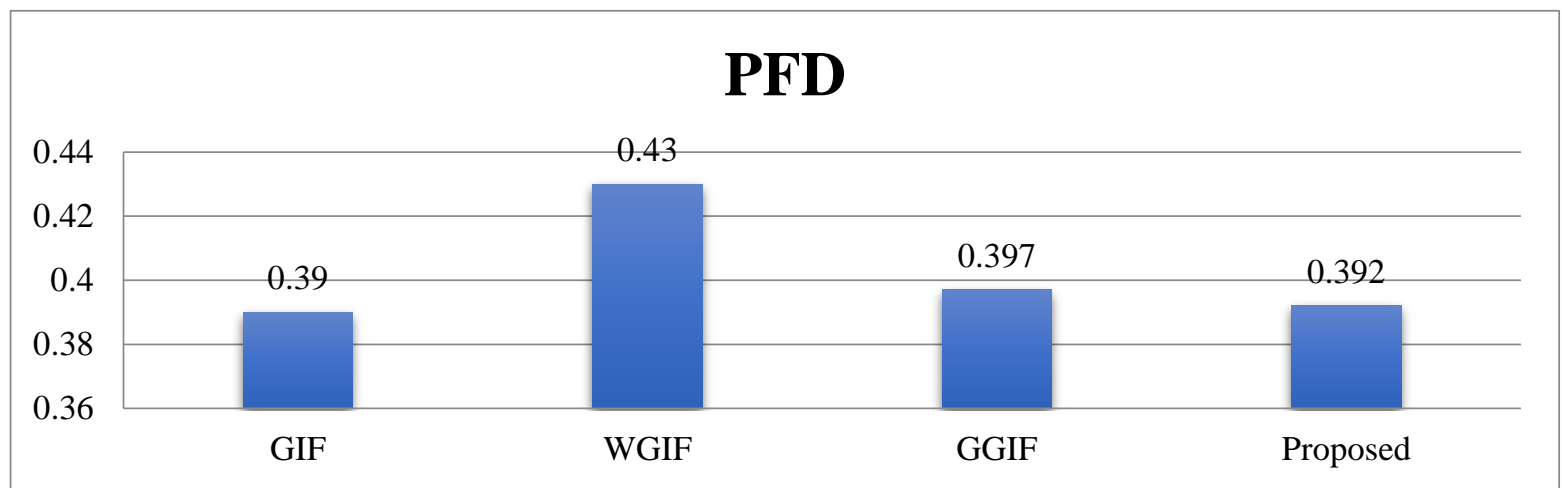

Fig. 5.4 Input Image 2 PFD output

In fig. 5.4, the fine structure is good in proposed and the PFD is lowest, but GIF is lower and color structure does not look realistic as per image and output as shown in fig. 5.5.

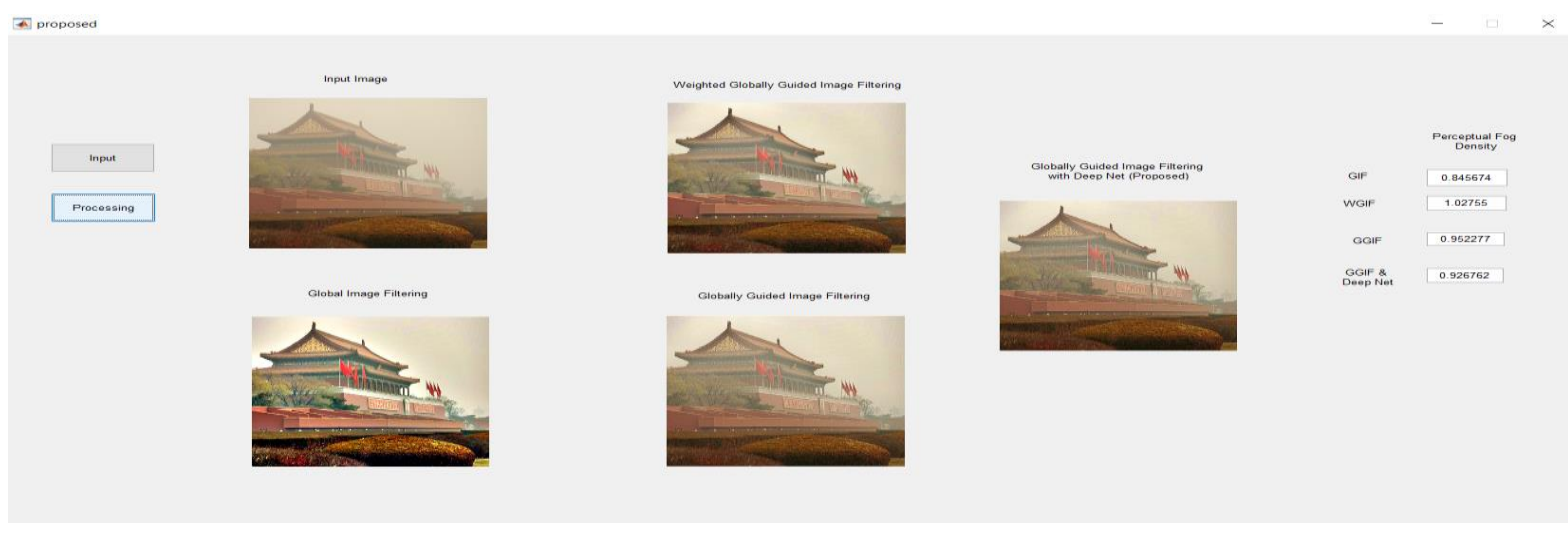

Fig. 5.5 Input Image 3 Output

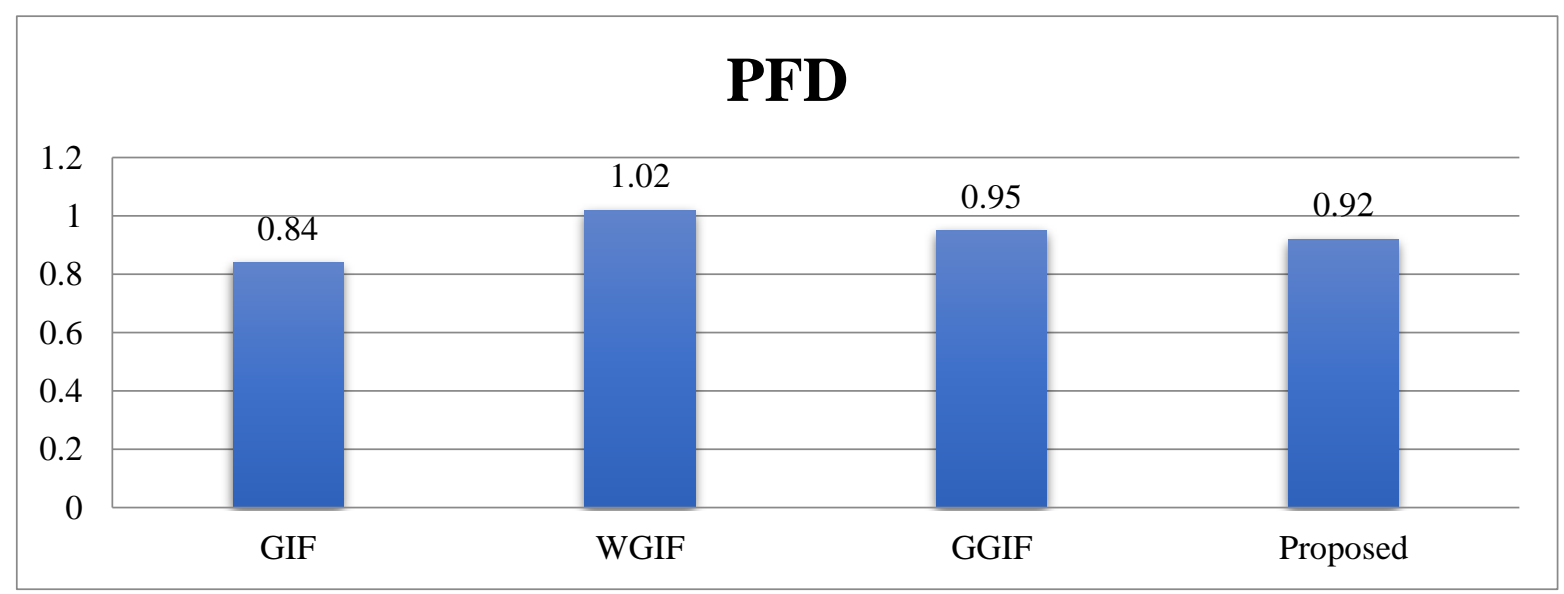

Fig. 5.6 Input Image 3 PFD Output

DOI Number: https://doi.org/10.30780/specialissue-ICACCG2020/043

Paper Id: IJTRS-ICACCG2020-043

pg. 64

@2017, IJTRS All Right Reserved, www.ijtrs.com 
In fig. 5.6, the image is good haze free in proposed algorithm and the PFD is lowest as shown in fig. 5.7.

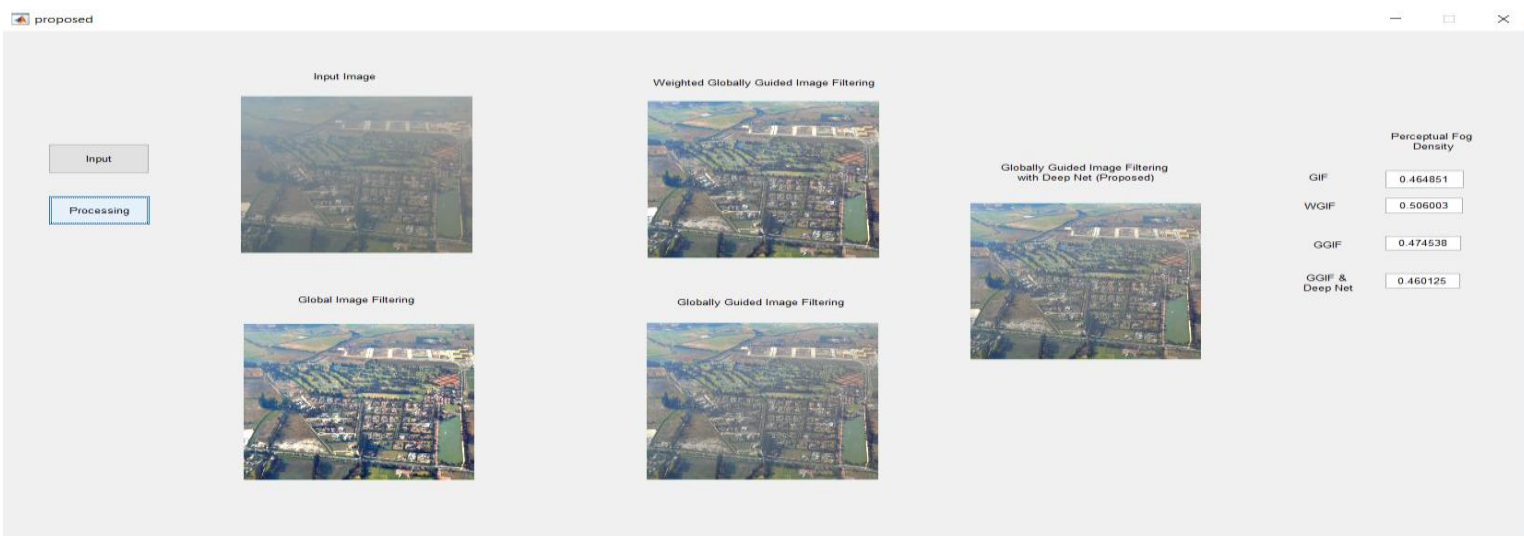

Fig. 5.7 Input Image 4 Output

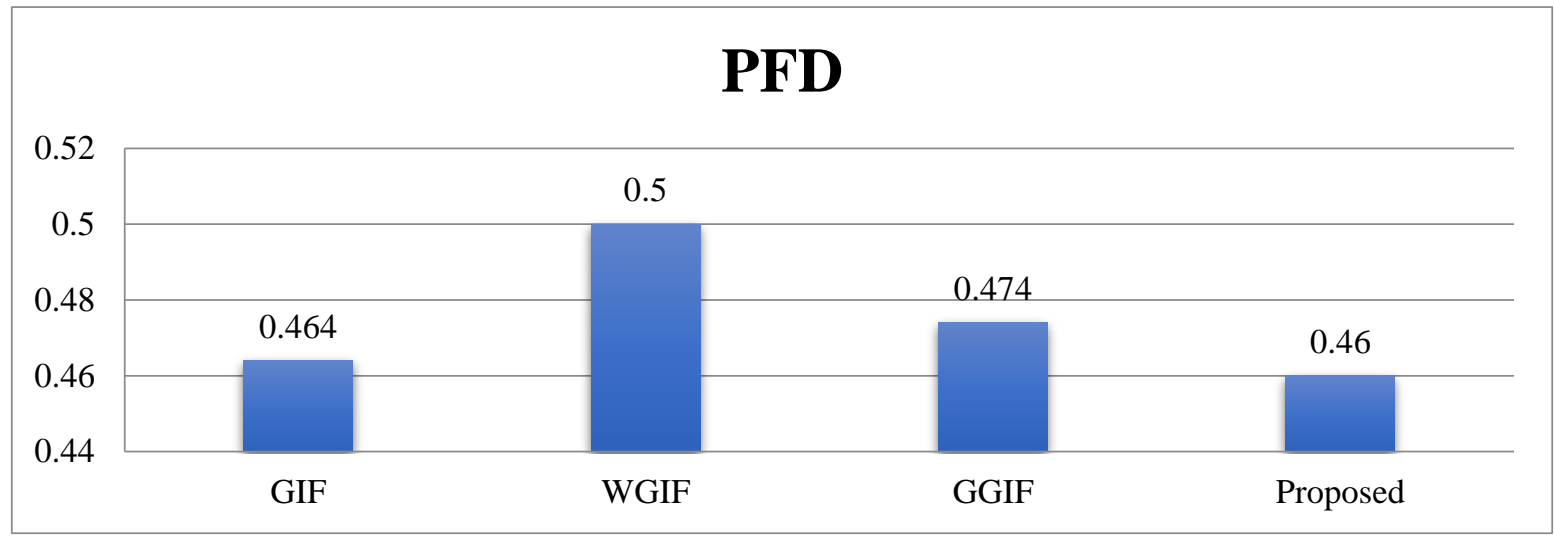

Fig. 5.8 Input Image 4 PFD Output

In fig. 5.9, the image is enhanced in proposed algorithm, and the PFD is lowest shown in fig. 5.10.

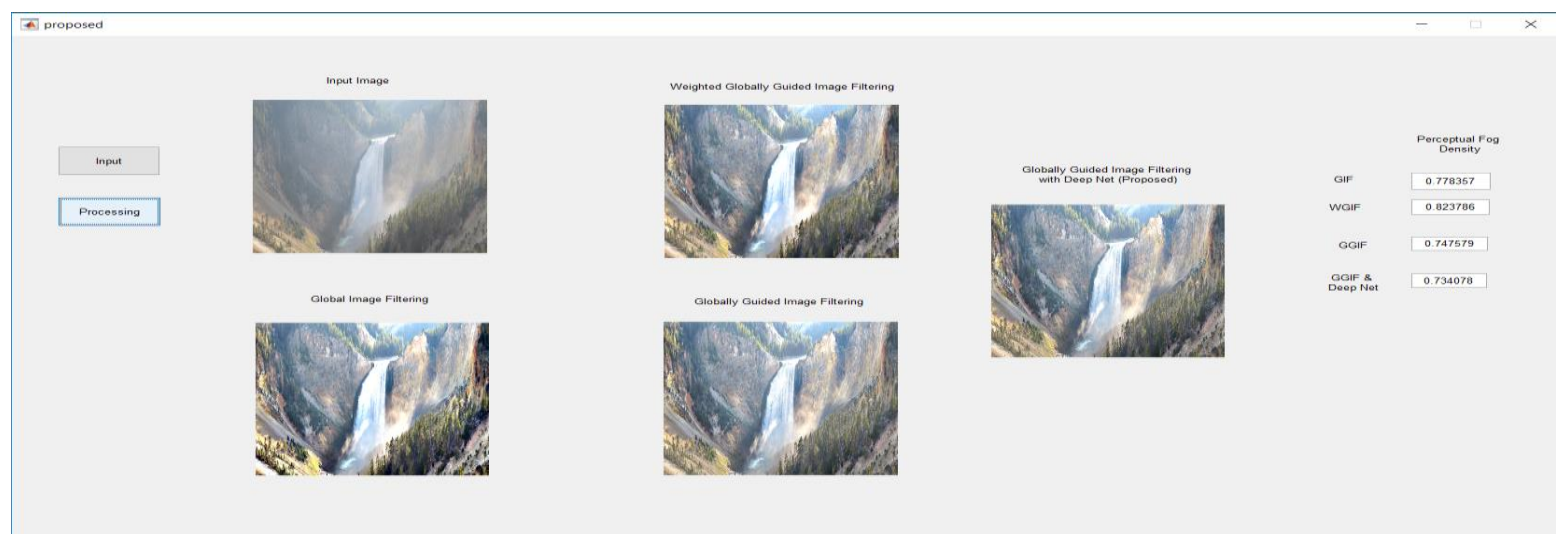

Fig. 5.9 Input Image 5 Output

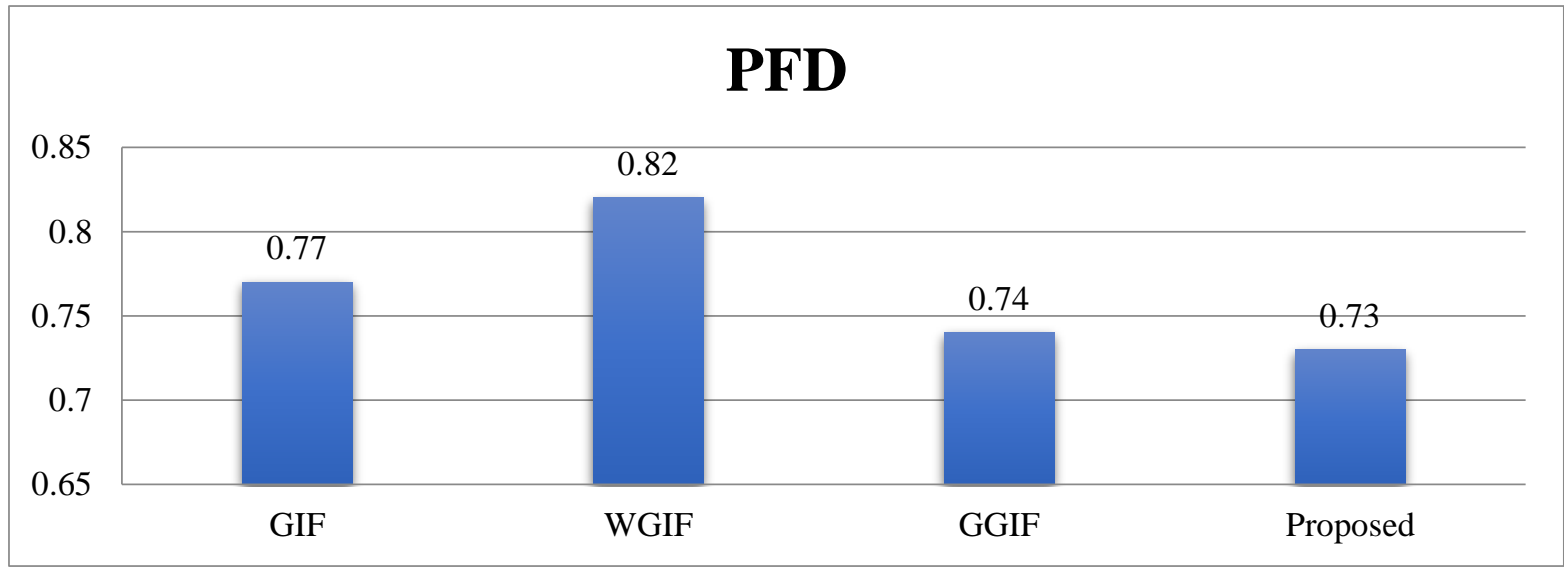

Fig. 5.10 Input image 5 PFD output

In fig. 5.11, the traffic is best enhanced in proposed with proper edges and colors and the PFD is lowest as shown in fig. 5.12 .

DOI Number: https://doi.org/10.30780/specialissue-ICACCG2020/043 

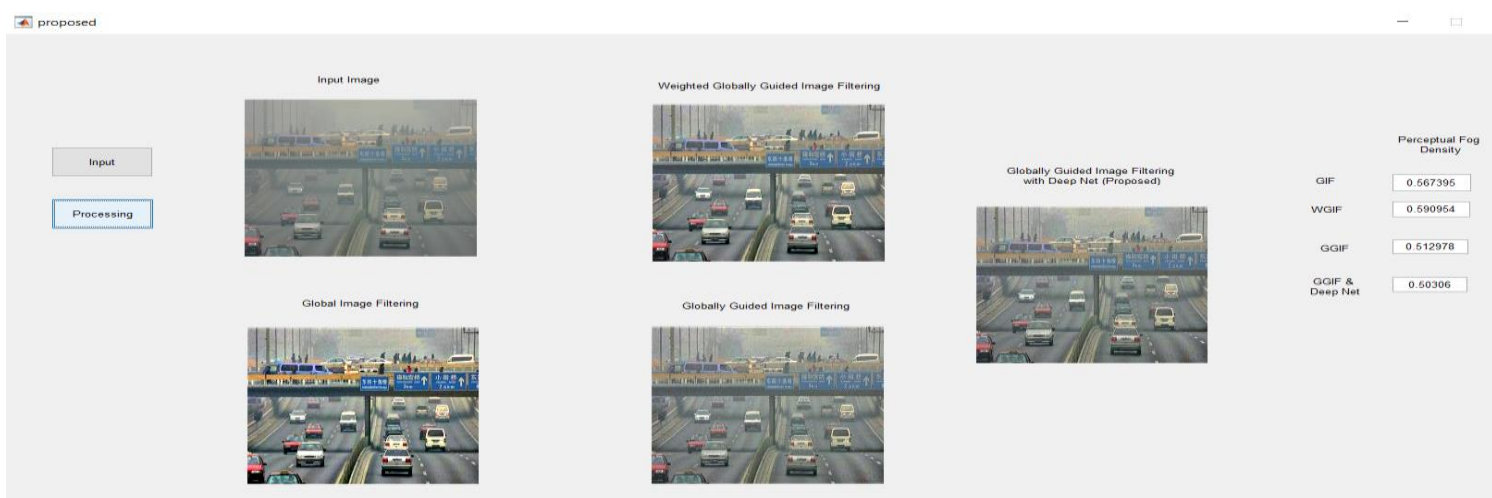

Fig. 5.11 Input Image 6 Output

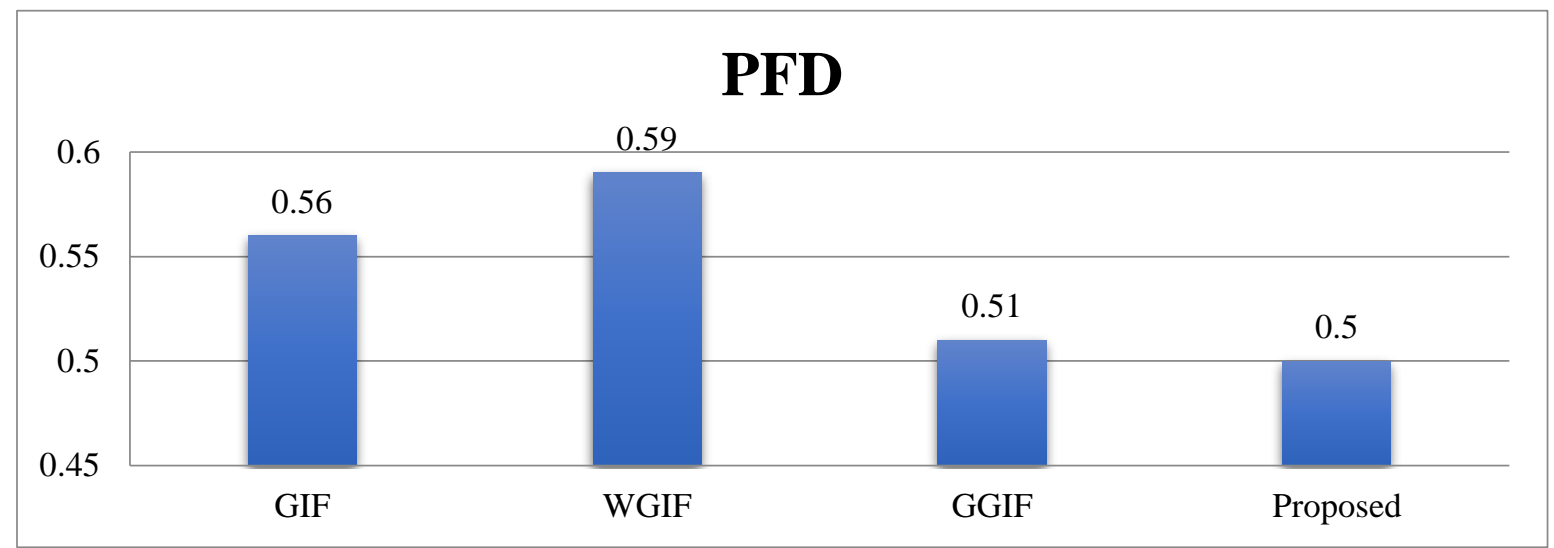

Fig. 5.12 Input Image 6 PFD Output

In fig. 5.13, the indoor image is enhanced and haze free in proposed algorithm and the PFD is lowest as shown in fig. 5.14 .

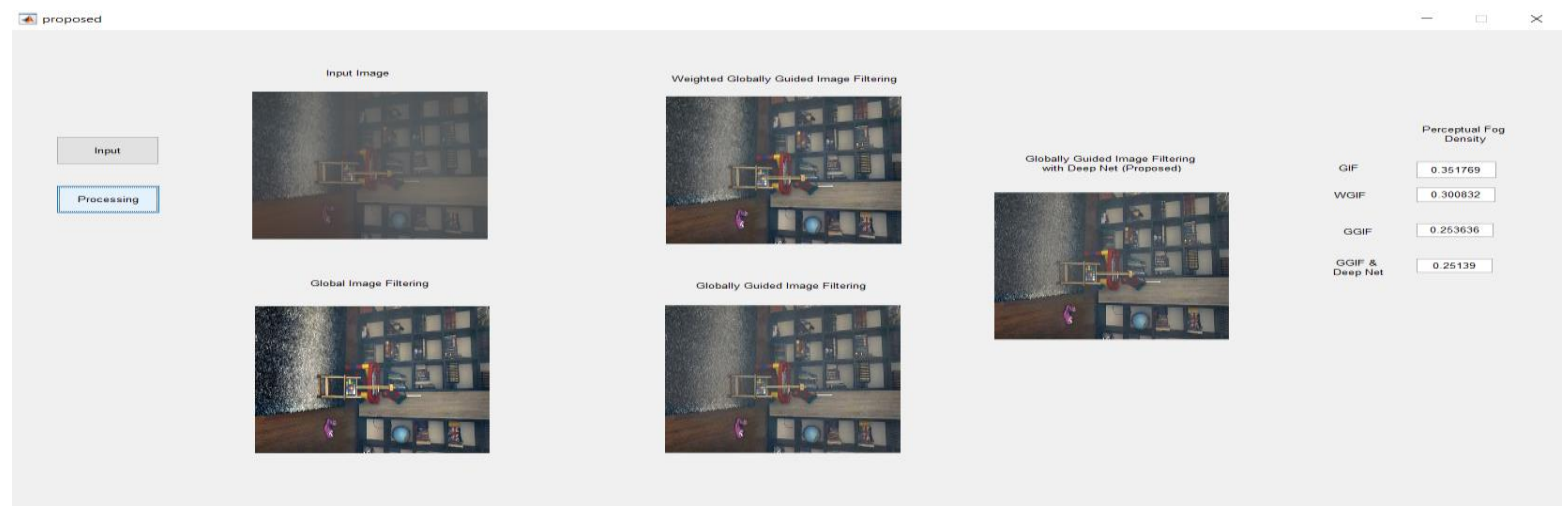

Fig. 5.13 Input Image 7 Output

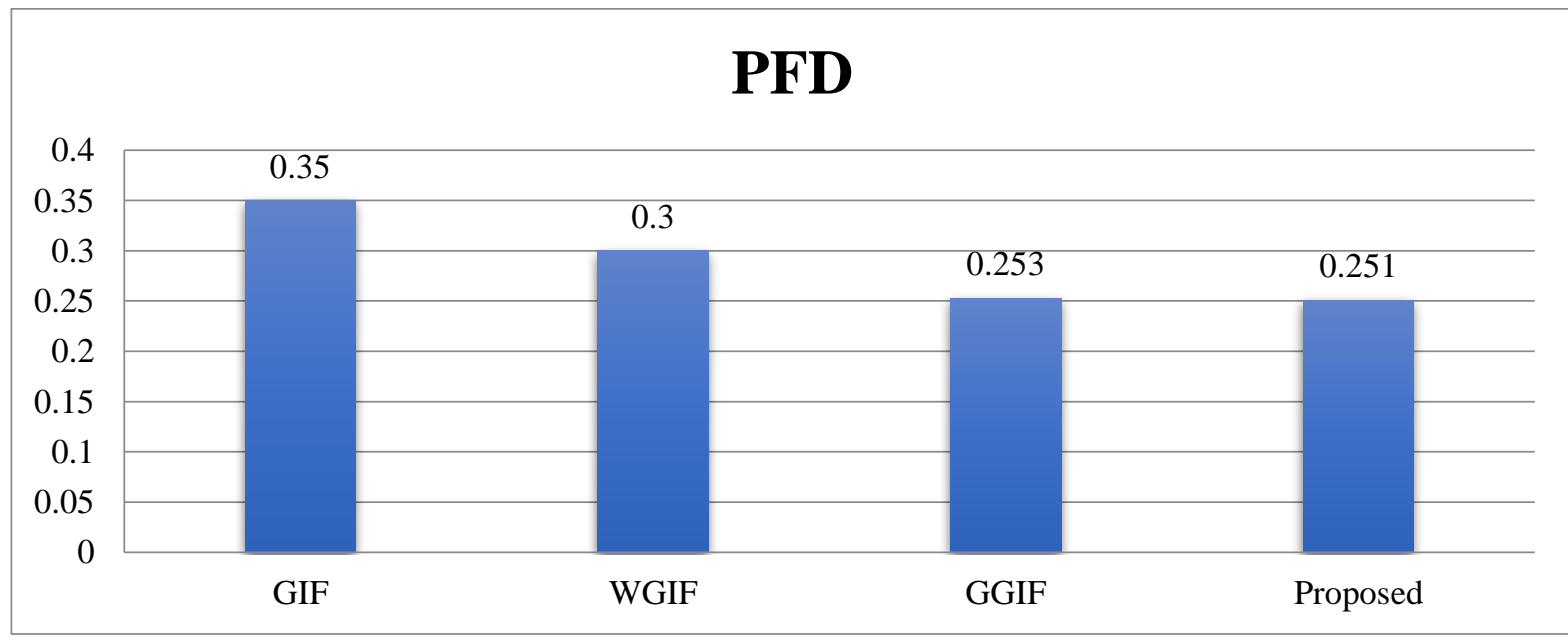

Fig. 5.14 Input Image 7 PFD Output

In fig. 5.15, the image for indoor office is good in proposed and the PFD is lowest as shown in fig. 5.16.

DOI Number: https://doi.org/10.30780/specialissue-ICACCG2020/043

Paper Id: IJTRS-ICACCG2020-043

pg. 66

@2017, IJTRS All Right Reserved, www.ijtrs.com 

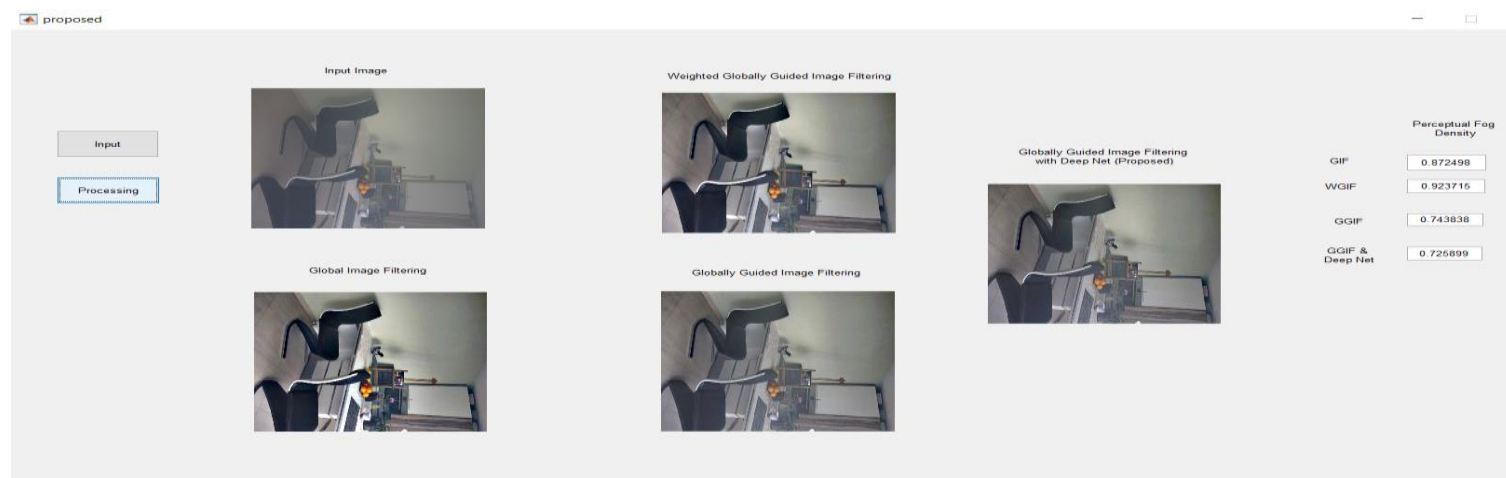

Fig. 5.15 Input Image 8 Output

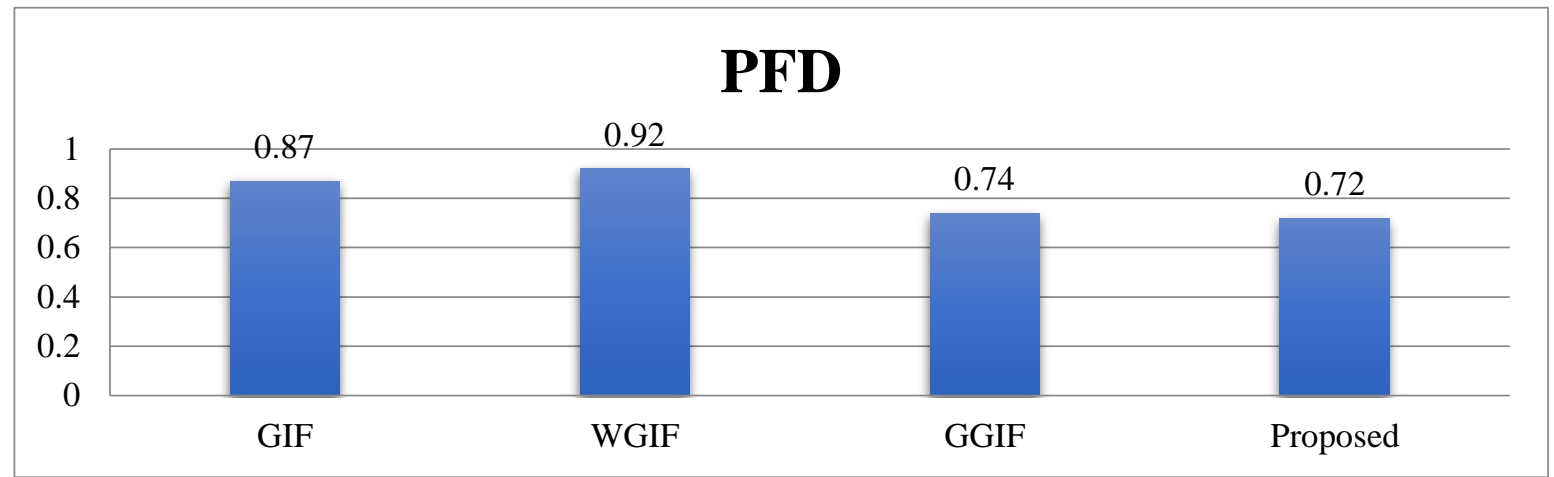

Fig. 5.16 Input Image 8 PFD Output

In all the images, the benefit of the proposed technique is shown and verified by output results.

\section{CONCLUSION}

Pictures caught in dim or foggy climate conditions can be truly corrupted by dissipating of barometrical particles, which diminishes the difference, changes the shading, and makes the object highlights hard to distinguish by human vision and by a few open air PC vision frameworks. Along these lines picture dehazing is a significant issue and has been generally looked into in the field of PC vision. The hazy images are improved by removal of haze, edge preserving, fine structure checking and realistic color matching is done to improve the image characteristics using deep learning neural network and an existing technique known as the globally guided image filtering technique. Machine learning is important part of image processing and several techniques are made to improve the hazy images. The main parameter of consideration is perceptual fog density which is improved in the proposed algorithm. This software is made on MATLAB environment, and its able to run any size and features and can be made haze free with utmost accuracy and color structures are preserved.

\section{REFERENCE}

[1] Zhengguo Li "Single Image De-Hazing Using Globally Guided Image Filtering" IEEE Transactions on Image Processing, Vol. 27, No. 1, January 2018.

[2] Dengyin Zhang, TY - JOUR, TI - FAOD-Net: A Fast AOD-Net for Dehazing Single Image, 2020/02/24, 10.1155/2020/4945214, https://doi.org/10.1155/2020/4945214

[3] He Zhang Vishwanath Sindagi Vishal M. Patel” Multi-scale Single Image Dehazing using Perceptual Pyramid Deep Network” InIm-age Processing (ICIP), 2016 IEEE International Conferenceon, pages 22262230. IEEE, 2016.

[4] He Zhang et al., "Multi-scale Single Image Dehazing using Perceptual Pyramid Deep Network" 2016 IEEE International Conference on, pages 2226-2230. IEEE, 2016.

[5] Nidhi Sen et al., "Weighted Guided Image Filtering - A Survey "International Journal of Computer Applications (0975 - 8887) Volume 156 - No 10, December 2016

[6] Yan Hu et al., "Underwater Image Restoration Based on Convolutional Neural Network" Proceedings of Machine Learning Research 95:296-311, 2018.

[7] Ranjan Mondal et al., "Image Dehazing by Joint Estimation of Transmittance and Airlight using Bi-Directional Consistency Loss Minimized FCN" June 2018.

[8] Dong Yang et al., "Proximal Dehaze-Net: A Prior Learning-Based Deep Network for Single Image 
Dehazing” Transactions on Image Processing 27(1), 379-393 (2018).

[9] W. Uma et al., "Globally Guided Image Filtering Method for Single Image Haze Removal Using An Transmission Map" International Journal of Emerging Technology in Computer Science \& Electronics (IJETCSE) ISSN: 0976-1353 Volume 25 Issue 5 - APRIL 2018 (SPECIAL ISSUE).

[10] Weiqiang Chen et al., "Reconfiguration of NPC Multilevel Inverters to Mitigate Short Circuit Faults Using Back-to-Back Switches” VOL. 3, NO.1, MARCH 2018.

[11] Wenqi Ren et al., "Gated Fusion Network for Single Image Dehazing” Single image dehazing by multiscale fusion. TIP, 22(8):3271-3282, 2013. 2, 4.

[12] Zhengguo Li et al., "Weighted Guided Image Filtering” IEEE Transactions On Image Processing, Vol. 24, No. 1, January 2015.

[13] Zhengguo Li et al., "Reconfiguration of NPC Multilevel Inverters to Mitigate Short Circuit Faults Using Back-to-Back Switches” pp. 3888-3901, Nov. 2015.

[14] F. Ragini Felicia Suruti et al., "Survey on Various Dehazing Techniques" IJCSMC, Vol. 5, Issue. 2, February 2016,pg.218 - 223.

[15] He Zhang et al., "Multi-scale Single Image Dehazing using Perceptual Pyramid Deep Network" 2016 IEEE International Conference on, pages 2226-2230. IEEE, 2016.

[16] Nidhi Sen et al., "Weighted Guided Image Filtering - A Survey "International Journal of Computer Applications (0975 - 8887) Volume 156 - No 10, December 2016.

[17] Yan Hu et al., "Underwater Image Restoration Based on Convolutional Neural Network" Proceedings of Machine Learning Research 95:296-311, 2018.

[18] Ranjan Mondal et al. "Image Dehazing by Joint Estimation of Transmittance and Airlight using Bi-Directional Consistency Loss Minimized FCN” June 2018.

[19] Dong Yang et al., "Proximal Dehaze-Net: A Prior Learning-Based Deep Network for Single Image Dehazing” Transactions on Image Processing 27(1), 379-393 (2018).

[20] W. Uma et al., "Globally Guided Image Filtering Method for Single Image Haze Removal Using an Transmission Map" International Journal of Emerging Technology in Computer Science \& Electronics (IJETCSE) ISSN: 0976-1353 Volume 25 Issue 5 - APRIL 2018 (SPECIAL ISSUE). 